\title{
Estudo Matemático dos fatores epigenéticos relacionados ao tumor de Wilms em crianças: modelagem e simulações
}

\author{
Marina Lima ${ }^{1}$ \\ DMA - IMECC - UNICAMP, Campinas, SP \\ Fernanda W. Picerni ${ }^{2}$ \\ DMA - IMECC - UNICAMP, Campinas, SP \\ Gislaine de O. Queiroz ${ }^{3}$ \\ DMA - IMECC - UNICAMP, Campinas, SP \\ Jaqueline T. Teixeira ${ }^{4}$ \\ DMA - IMECC - UNICAMP, Campinas, SP \\ Lucas C. de Barros ${ }^{5}$ \\ DMA - IMECC - UNICAMP, Campinas, SP \\ Pedro N. Ramos ${ }^{6}$ \\ DMA - IMECC - UNICAMP, Campinas, SP \\ Ravine T. Wenningkamp ${ }^{7}$ \\ DM - UFSM, Santa Maria, RS \\ Vinícius F. Fernandes ${ }^{8}$ \\ DMA - IMECC - UNICAMP, Campinas, SP \\ João Frederico de Azevedo Meyer ${ }^{9}$ \\ DMA - IMECC - UNICAMP, Campinas, SP \\ Pedro Víctor Carvalho ${ }^{10}$ \\ IB - UNICAMP, Campinas, SP
}

\begin{abstract}
Resumo. Embora, anualmente, cerca de 12 mil crianças e adolescentes sejam diagnosticadas com câncer no Brasil, ainda não há um consenso sobre a origem do câncer infantil, mas existem provas de que ele é causado por alterações em células embrionárias, as quais podem ser resultantes de fatores externos de exposição materna ou paterna, o que torna fundamental o estudo desses fatores, em busca de um diagnóstico precoce, visando o aumento das chances de cura. Neste trabalho, estudamos o tumor de Wilms, o qual é um tipo de câncer comum em crianças, construímos um modelo matemático e realizamos simulações para diferentes cenários de tratamento oncológico, destacando a importância dos fatores externos na eliminação ou manutenção das células cancerígenas, o que é fundamental para a determinação da eficiência do processo terapêutico.
\end{abstract}

Palavras-chave. Modelo Epigenético, Simulação do Tumor de Wilms, Modelagem Matemática

\footnotetext{
${ }^{1}$ marina@ime.unicamp.br

2 wohlersfe@gmail.com

3 g155579@dac.unicamp.br

4 jaquelinetakekoshi@gmail.com

${ }^{5}$ lucascavalcantedebarros@gmail.com

${ }^{6}$ p185812@dac.unicamp.br

7 ravinetaissw@gmail.com

8v157510@dac.unicamp.br

9joni@ime.unicamp.br

${ }^{10}$ pedrovictorcarvalho@icloud.com
} 


\section{Introdução}

O tumor de Wilms, também conhecido como nefroblastoma, corresponde a cerca de 95\% de todos os cânceres renais e $6 \%$ dos casos de câncer infantis (abaixo dos 15 anos) [1, 7, 12]. Se apresenta como um tumor embrionário maligno com origem em células precursoras dos rins. Apesar de robustos avanços no diagnóstico e tratamento, o que promove um aumento nas taxas de cura e aumento da sobrevida em 5 anos em mais de $85 \%$ dos casos, os efeitos colaterais e risco a curto e longo prazo são altos para os pacientes durante o tratamento $[2,8]$.

Dessa forma, precisamos compreender melhor as possíveis variáveis de etiologia e patologia do tumor de Wilms, o que pode contribuir positivamente para a qualidade de vida das crianças que venham a ser diagnosticadas com esse tipo de câncer. Entretanto, para analisarmos a causa do tumor de Wilms em crianças, devemos investigar qual o fenômeno precursor do mesmo nos pais, o que é feito através do estudo dos fatores externos capazes de causar a mutação genética, que irá resultar na formação das células tumorais nas crianças. Esse tipo de estudo só é possível devido ao desenvolvimento da Epigenética, que é uma área da Biologia que abrange estudos sobre alterações em um gene, as quais podem ser herdáveis [7]. Tais alterações podem ocorrer durante toda a vida, sendo elas metilação do DNA (ácido desoxirribonucleico), modificação nas histonas ou expressão de RNA (ácido ribonucleico) não codificantes.

Doenças complexas são causadas por fatores ambientais e genéticos. A exemplo, temos obesidade, diabetes tipo II e hipertensão, as quais precisam apresentar os dois fatores para se desenvolver, podendo assim, por meio de mudanças no ambiente (principalmente hábitos de vida), evitar seu surgimento no indivíduo [11]. Não se sabe se todas as doenças complexas podem ser evitadas ao se modificar os fatores ambientais, no entanto, estamos interessados em discorrer sobre alguns fatores que podem resultar em carcinomas, especialmente no Tumor de Wilms.

O presente trabalho consiste em uma investigação epigenética sobre o aparecimento do Tumor de Wilms em crianças, uma vez que fatores externos podem ser responsáveis por desregulação dos genes supressores de tumores e por outras alterações gênicas que cooperam com o desenvolvimento e progressão do câncer. O trabalho é resultante de uma parceria entre o Centro Boldrini, um centro referência no tratamento de cânceres em crianças, localizado em Campinas - SP, e o Instituto de Matemática, Estatística e Computação Científica (IMECC - UNICAMP), de maneira a integrar a Matemática Aplicada e a Oncologia e construir modelos capazes de simular e predizer a relevância de fatores epigenéticos no desenvolvimento do tumor de Wilms e na busca de uma resposta para a prevenção do mesmo.

\section{Metodologia}

O tumor de Wilms é um tipo de tumor maligno que se inicia no rim, podendo atingir apenas um ou os dois rins [5]. O tumor possui maior incidência na primeira infância e raramente acomete adultos. O gene do tumor de Wilms 1 (WT1) está localizado no braço curto do cromossomo 11 (11p13) e as mutações germinativas desse gene têm sido encontradas em cerca de $2 \%$ das crianças que apresentam esse carcinoma [4].

A ideia de um modelo epigenético não é contradizer o modelo genético, mas sim complementálo, o que inclui, considerar que alterações epigenéticas podem contribuir na carcinogênese e crescimento tumoral. Dados os impactos que o ambiente apresenta em estudos que relacionam o meio e metilação nas células, é necessário ter atenção em alguns componentes epigenéticos, como meio ambiente e substâncias alimentícias carcinógenas, mas eles ainda precisam ser estudados [3].

Alguns estudos epidemiológicos anteriores sugeriram que a exposição a pesticidas no útero e durante a primeira infância pode aumentar o risco de desenvolvimento de câncer infantil, incluindo o tumor de Wilms, leucemia infantil, neuroblastoma, entre outros [10]. Além disso, a exposição 
ocupacional dos pais aos agrotóxicos, produtos gráficos, tintas, metais pesados, entre outros, contribuem para a carga cumulativa das crianças, o que resulta em uma maior incidência de câncer infantil [6]. Entretanto, uma questão importante é o tempo de exposição necessário para que a criança desenvolva este tipo de câncer, atribuído à fatores epigenéticos.

Para o desenvolvimento do nosso trabalho, inicialmente analisamos os dados sobre câncer renal no Brasil, de 1990 a 2015, uma vez que esse é um tipo de tumor renal comum na infância e pode acometer um ou ambos os rins [5]. Tais dados estão apresentados na Figura 1.

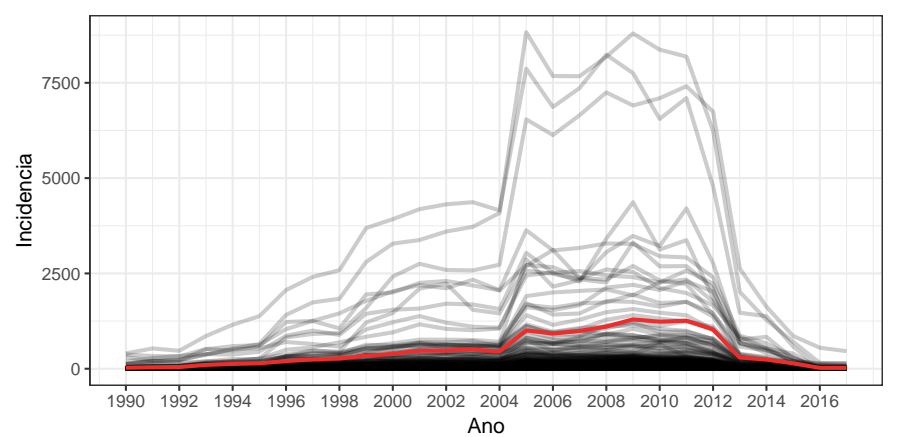

Figura 1: Incidência de câncer no Brasil de 1990 a 2015. Os dados sobre o tumor de Wilms estão assinalados em vermelho. Dados obtidos em [5].

Na Figura 1 podemos observar que, embora o tumor de Wilms não seja o tipo mais comum de câncer, o mesmo possui uma incidência elevada, sendo importante o estudo do mesmo. Ademais, o fato que mais atraiu atenção, foi quando analisamos a distribuição da incidência do tumor de Wilms pela idade dos indivíduos, como apresentado nos gráficos da Figura 2.

Analisando os gráficos da Figura 2, observamos que o tumor de Wilms apresenta uma incidência considerável em crianças de 0 a 7 anos, seguido por uma queda e por um aumento na proporção dos casos na idade adulta. Esse tipo de comportamento nos sugere que fatores externos estão afetando os pais e resultando no acometimento do feto (antes mesmo da formação). Fato esse confirmado pela baixa incidência desse tipo de tumor em crianças com idade acima de 7 anos.

Baseado nessas informações, construímos um modelo matemático que relaciona as células normais, intermediárias e cancerígenas com os fatores ambientais e analisamos a dinâmica da doença em vários cenários possíveis. O modelo e os resultados são apresentados nas seções 3 e 4 .

\section{Modelagem Matemática}

Baseado nas hipóteses apresentadas na seção 2, apresentamos um modelo que descreve o processo envolvido no crescimento das células, incluindo a influência causada pelo fator epigenético introduzido nas equações. O modelo é baseado na seguinte dinâmica apresentada na Figura 3.

Dessa forma, temos as seguintes variáveis de estado: $N(t)$ são as células saudáveis, $I(t)$ são as células intermediárias e $C(t)$ são as células cancerígenas, todas analisadas no tempo $t$, e os parâmetros: $\alpha_{N}$ : taxa de mutação das células normais, se tornando intermediárias; $\alpha_{N H}$ : taxa de mutação das células intermediárias em cancerígenas, induzidas por fatores hereditários; $\alpha_{N E}$ : taxa de mutação das células intermediárias em cancerígenas, induzidas por fatores externos; $\alpha_{I}$ : taxa de células intermediárias que se tornam cancerígenas $\left(\alpha_{I}=\alpha_{N H}+\alpha_{N E}\right) ; p$ taxa de apoptose (morte programada) celular devido ao tratamento; $\mu$ : taxa das células cancerígenas que tornam-se intermediárias; $\lambda_{i}$ : taxa de crescimento intrínseco de cada classe de células, sendo $i=N, I, C$; 


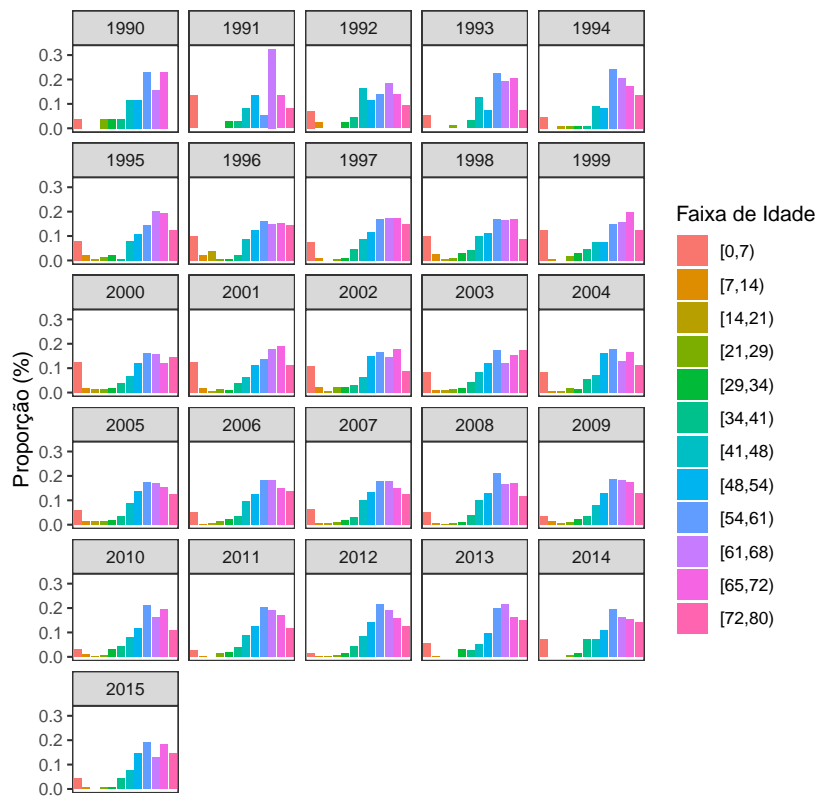

Figura 2: Proporção de casos do tumor de Wilms, em relação à idade do indivíduo, de 1990 a 2015. Dados obtidos em [5].

$\rho$ : taxa de competição entre as células normais e as intermediárias; $\nu$ : taxa de competição entre as células normais e as cancerígenas; $\delta$ : taxa de competição entre as células intermediárias e as cancerígenas; $\sigma$ : taxa de células intermediárias que se tornam normais; $K$ : capacidade de suporte, sendo a mesma para as três classes de células, uma vez que o tumor é localizado nos rins e elas competem pelo mesmo espaço e pelas mesmas condições.

Ademais, incluímos no modelo uma situação na qual a inibição e os estímulos angiogênicos estão em equilíbrio antes do tratamento iniciar e que o tratamento é feito seguindo a hipótese log-kill, em que há a eliminação das células tumorais em proporção constante, a cada infusão do agente quimioterápico [9]. Então, denotamos $Q(t)$ a quantidade do agente quimioterápico e, dessa forma, podemos construir o seguinte modelo de equações diferenciais ordinárias:

$$
\begin{aligned}
\frac{\mathrm{d} N(t)}{\mathrm{d} t} & =\lambda_{N} N(t)\left(1-\frac{N(t)+\rho I(t)+\nu C(t)}{K}\right)-\alpha_{N} N(t)+\sigma I(t)-\frac{a_{1} N(t) Q(t)}{b_{1}+Q(t)} \\
\frac{\mathrm{d} I(t)}{\mathrm{d} t} & =\lambda_{I} I(t)\left(1-\frac{I(t)+\rho N(t)+\delta C(t)}{K}\right)+\alpha_{N} N(t)-\alpha_{I} I(t)+\mu(1-p) C(t)-\frac{a_{1} I(t) Q(t)}{b_{1}+Q(t)} \\
\frac{\mathrm{d} C(t)}{\mathrm{d} t} & =\lambda_{C} C(t)\left(1-\frac{C(t)+\nu N(t)+\delta I(t)}{K}\right)+\alpha_{I} I(t)-\mu C(t)-\frac{a_{2} C(t) Q(t)}{b_{2}+Q(t)} \\
\frac{\mathrm{d} Q(t)}{\mathrm{d} t} & =q(t)-\lambda Q(t)
\end{aligned}
$$

em que, além dos parâmetros descritos anteriormente, temos $a_{1}$ : taxa de mortalidade das células normais e intermediárias em decorrência do tratamento, uma vez que a quimioterapia também afeta essas células; $a_{2}$ : taxa de tratamento das células tumorais; $b_{1}$ e $b_{2}$ : saturação do efeito resposta a droga; $q(t)$ : taxa de administração da droga; e $\lambda$ : taxa de decaimento de um dado agente quimioterápico ciclo-específico. A interação entre as células normais, intermediárias e tumorais é dada por Lotka-Volterra, pois essa é uma circunstância na qual o modelo logístico é o mais 


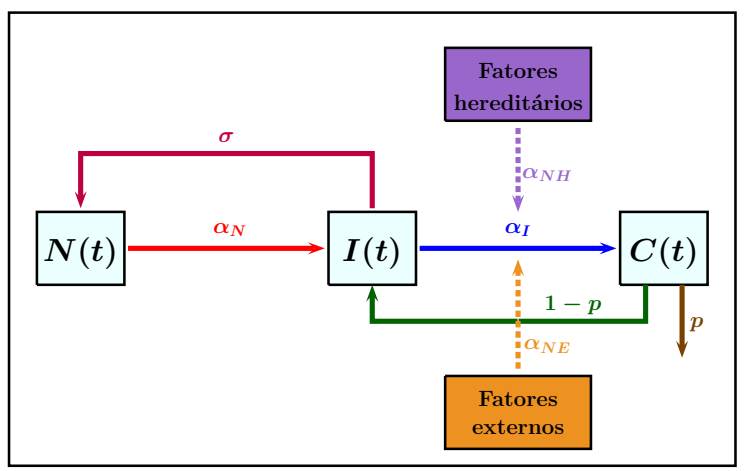

Figura 3: Diagrama que representa a dinâmica celular proposta.

adequado para o ajuste de dados de crescimento para humanos [9].

\section{Resultados e Discussão}

Baseado no conjunto composto pelas equações (1) - (4), realizamos simulações numéricas para diversos cenários e analisamos os gráficos oriundo dos mesmos. Para tais simulações, utilizamos o Wolfram Mathematica ${ }^{\circledR} 12.0$, o qual utiliza o método de Runge-Kutta de quarta ordem para a resolução dos sistemas. Utilizamos somente uma administração quimioterápica, com uma dose elevada [9], e simulamos dois cenários: no primeiro há a eliminação das células cancerígenas (Figura 4) e, no segundo, a eliminação das células normais (Figura 5).

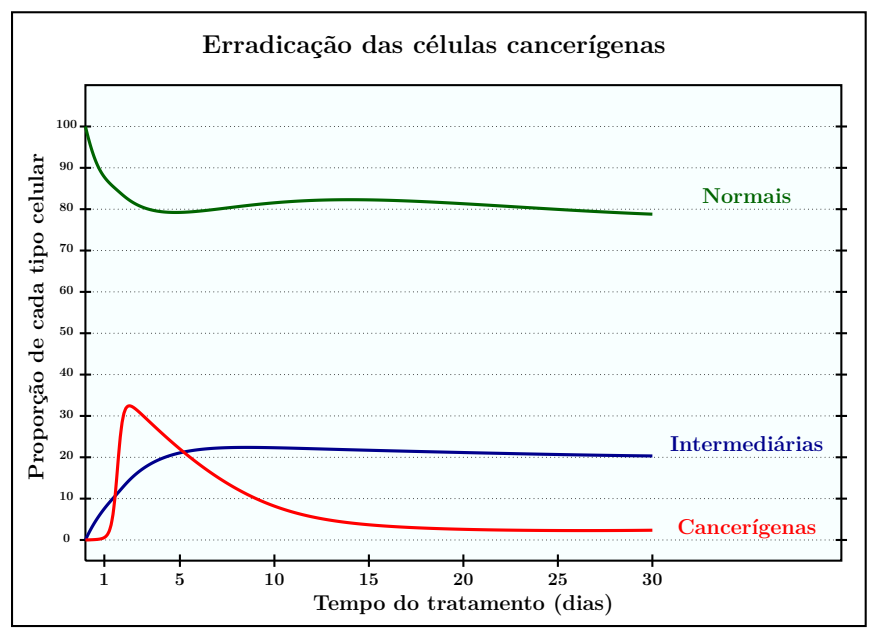

Figura 4: Comportamento das células normais, intermediárias e cancerígenas, mediante administração de quimioterápico em dose única.

O gráfico da Figura 4 representa o comportamento das células dos rins mediante a aplicação de uma dose de quimioterápico. Como parâmetro de simulação, utilizamos um valor baixo para o parâmetro $\alpha_{N E}$, que representa a influência externa no comportamento celular e, no modelo, é responsável pela conversão de células intermediárias em cancerígenas. Como resultado, temos 
uma queda na proporção de células normais e um aumento na proporção de intermediárias e de cancerígenas, mas como há uma baixa taxa de mutação de células intermediárias em cancerígenas, o tratamento é suficiente para a eliminação das mesmas, o que resulta na coexistência de células normais e intermediárias, ou seja, o câncer é eliminado.

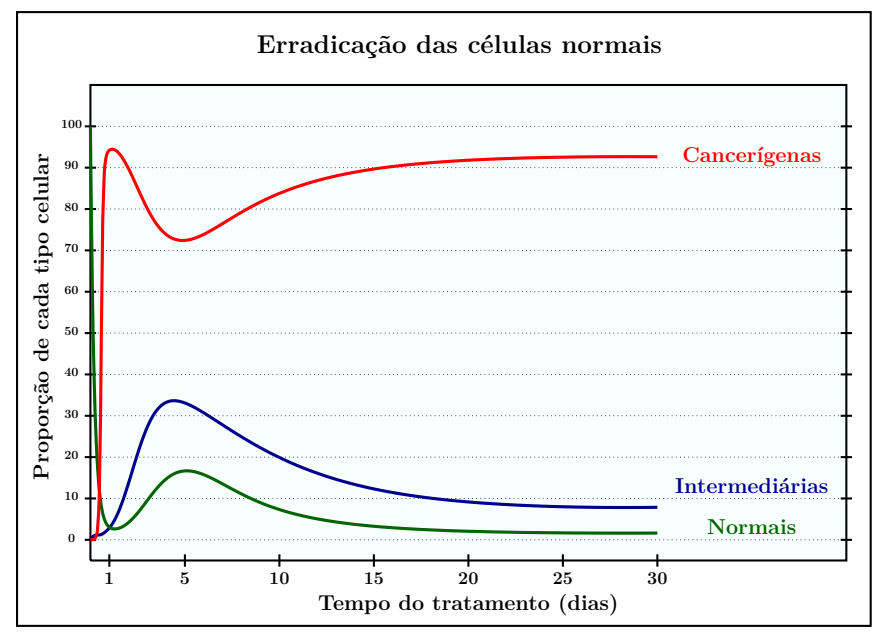

Figura 5: Comportamento das células normais, intermediárias e cancerígenas, mediante administração de quimioterápico em dose única.

O gráfico da Figura 5 representa uma situação oposta ao gráfico da Figura 4. Nesse caso, temos um valor alto para a influência externa na mutação das células intermediárias, ou seja, para o parâmetro $\alpha_{N E}$. Dessa forma, observamos um rápido crescimento da proporção das células cancerígenas, seguido por uma queda do mesmo e, consequentemente, um aumento na proporção das células normais e intermediárias. Essa diminuição é resultado do tratamento quimioterápico aplicado (em dose única), mas em seguida, observamos um aumento das células cancerígenas, uma vez que há uma influência externa significativa na conversão de células intermediárias em cancerígenas. Como consequência, temos a eliminação das células normais e a coexistência das células intermediárias e de cancerígenas, o que indica a metástase do órgão analisado.

\section{Considerações Finais}

Os resultados do presente estudo deixaram evidente que os fatores externos são fundamentais para o crescimento e eliminação do tumor de Wilms, como tínhamos por objetivo. Não analisamos a aplicação de mais doses de quimioterapia, nem o efeito da hipótese log-kill para os fracassos e sucessos clínicos de protocolos de tratamento do câncer. Outras simulações e análises serão feitas nos próximos trabalhos e, dessa forma, podemos elucidar de forma mais clara os efeitos de cada um dos fatores externos e de como a quimioterapia pode atuar como coadjuvante na inibição da influência dos mesmos nos processos de mutação celular que levam ao câncer. Ademais, estamos buscando mais informações qualitativas sobre os pacientes, como as regiões que eles residem, os hábitos, entre outras, o que é fundamental para um estudo epigenético mais detalhado e para uma resposta mais precisa quanto à prevenção do câncer de Wilms e de outros cânceres infantis. 


\section{Referências}

[1] Bernstein, L., Linet, M., Smith, M. A. and Olshan, A. F. Renal tumors. In Cancer Incidence and Survival among Children and Adolescents: United States SEER Program 1975-1995. National Cancer Institute, pages 79-90, 1999.

[2] Chu, A., Heck, J. E., Ribeiro, K. B., Brennan, P., Boffetta, P., Buffler, P., and Hung, R. J. Wilms' tumour: a systematic review of risk factors and meta-analysis. Paediatric and perinatal epidemiology, 24(5):449-469, 2010. DOI: 10.1111/j.1365-3016.2010.01133.x.

[3] Feinberg, A. P. The epigenetics of cancer etiology, Seminars in cancer biology, 14: 427-432, 2004. DOI: 10.1016/j.semcancer.2004.06.005.

[4] Graac - Grupo de Apoio ao Adolescente e à Criança com Câncer. Tumor de Wilms, https://graacc.org.br/tumor-de-wilms/. Acesso em 11 de Fevereiro de 2020.

[5] INCA - Instituto Nacional de Câncer. Tumor de Wilms - versão para Profissionais de Saúde, https://www.inca.gov.br/tipos-de-cancer/cancer-infantojuvenil/tumor-dewilms/profissional-de-saude. Acesso em 13 de Fevereiro de 2020.

[6] Infante-Rivard, C. and Weichenthal, S. Pesticides and Childhood Cancer: An Update of Zahm and Ward's 1998 Review, Journal of Toxicology and Environmental Health, Taylor \& Francis, 10(1-2):81-99, 2007. DOI: 10.1080/10937400601034589.

[7] Little, J. Epidemiology of Childhood Cancer, Lyon: International Agency for Research on Cancer, 1999.

[8] Pastore, G., Znaor, A., Spreafico, F., Graf, N., Pritchard-Jones, K., Steliarova-Foucher, E. Malignant renal tumours incidence and survival in European children (1978-1997): report from the Automated Childhood Cancer Information Systemproject. European Journal of Cancer, 42:2103-2114, 2006. DOI: 10.1016/j.ejca.2006.05.010.

[9] Rodrigues, D. S., Pinho, S. T. R. e Mancera, P. F. A. Um modelo matemático em quimioterapia, TEMA, 13:1-12, 2012. DOI: 10.5540/tema.2012.013.01.0001 .

[10] Sharpe, C. R., Franco, E. L., De Camargo, B., Lopes, L. F., Barreto, J. H., Johnsson, R. R. and Mauad, M. A. Parental exposures to pesticides and risk of Wilms' tumor in Brazil, American journal of epidemiology, 141:210-217, 1995. DOI: 10.1093/oxfordjournals.aje.a117422.

[11] Stessman, H. A., Bernier, R. and Eichler, E. E. A genotype-first approach to defining the subtypes of a complex disease, Cell, Elsevier, 156(5):872-877, 2014. DOI: 10.1016/j.cell.2014.02.002.

[12] Stiller, C. A., Allen, M. B. and Eatock, E. M. Childhood cancer in Britain: the National Registry of Childhood Tumours and incidence rates 1978-1987, European Journal of Cancer 31:2028-2034, 1995. DOI: 10.1016/0959-8049(95)00428-9. 